\title{
Mixed Dialogue Control Defined by an Attribute Grammar
}

\author{
U. LÄMMEL, P. FORBRIG \\ Universität Rostock, Sektion Informatik \\ Albert-Einstein-Str. 21, Rostock, DDR - 2500
}

Attribute Grammars (AGs) have been used for the formal description and automated implementation of various programming languages. Some approaches have been made to use AGs for the definition and implementation of even dialogue-languages: [OLSEN83], [LÄMMEL85], [DANG89].

If we look at dialogue-languages we can infer that AGs support the definition and implementation of command languages only. On the other hand a good interface between user and computer requires different activities, at least command- as well as menu-driven interactions.

We propose to use an AG for the homogeneous definition and implementation of command- and menu-dialogs. First we discuss the generation of menus getting necessary information from the AG. Whereas in approaches like [DANG89] the author of the language has to describe the handling of menus using semantic functions we generate menus automatically. For this purpose we must know for which nonterminals menus have to be generated. Thus a trisection of the $A G$ defining a dialogue-language is suggested:

(1) The dialogue-grammar describing the control of a dialogue but not the structure of single commands.

(2) A set of nonterminals: For these nonterminals menus will be produced.

(3) A set of rules defining the structure of single commands. 
A small calculator will serve as an example:

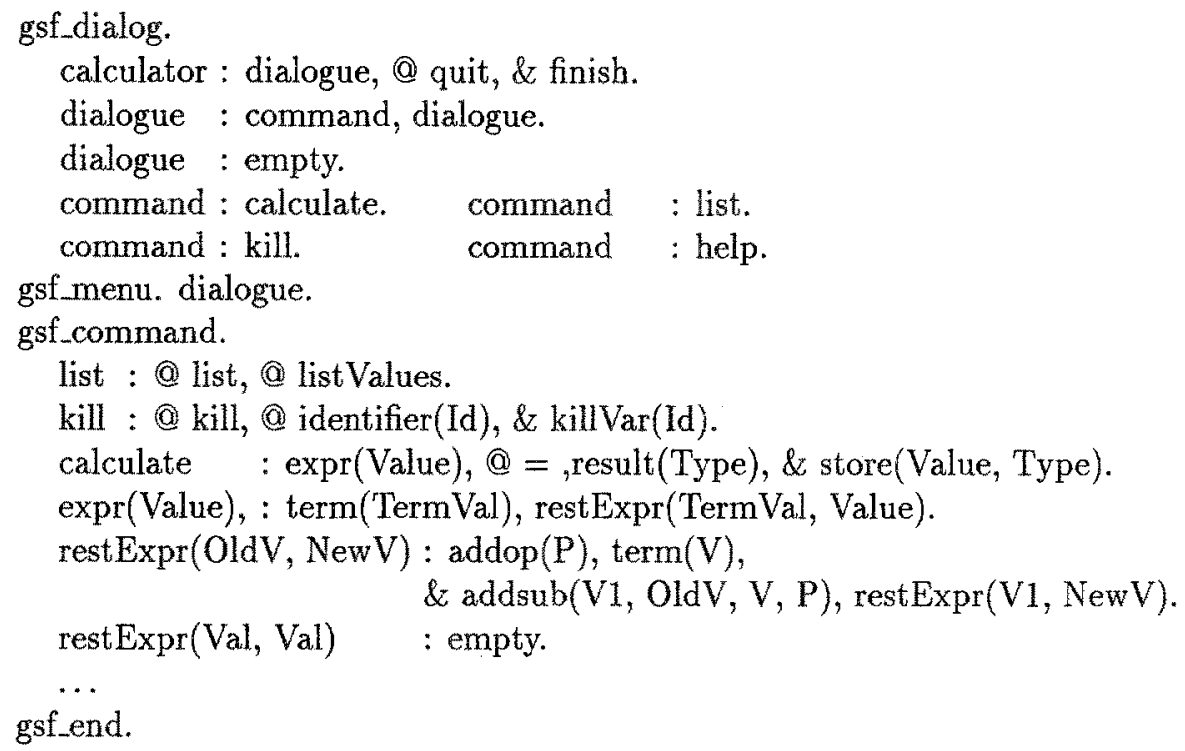

The ideas have been realized using a special form of an Attribute Grammar, Grammars of Syntactical Functions (GSF), and an implementation of this kind of AGs in PROLOG (Terminals and semantic functions are marked by the prefix operators @ and \& respectively).

Our approach to the construction of menus is based on the so called first- and followsets used in the theory of LL(1)-grammars. The set of menu options for a nonterminal $n$ menu (MG) consists of all first- and follow-elements of the nonterminal. Here elements can be terminals within the dialog-grammar or nonterminals for which menus have to be constructed, too, or nonterminals which are not further declared within the dialoggrammar. The later ones are nonterminals which represent complete commands further declared within the 'gsf_command' part. In our example we get the following set of options for the menu of the nonterminal 'dialogue':

$$
\text { \{list, kill, calculate, inf, quit\}. }
$$

The proposed method allows an easy definition of menuhierarchies too. The author of the language has to write down the set of nonterminals with menus only. The hierarchy is built automatically according to the formalism described above.

Having built all menus, the grammar can be executed. Executing this grammar in the conventional way using the underlying PROLOG-control leads to a recursive descent interpretation. Interactions, menu-choice or input of a command, are implemented as a mixed execution of rules of an AG. Sometimes the acceptence of an input is the main activity of the rule-interpreter and sometimes the presentation of several alternatives as well as the generation of sequences of terminals is performed. 
The evaluation of semantic functions is not fixed by the implementation of the GSF. Different definitions of the operator $\&$ lead to different evaluation strategies.

For dialogues we propose to collect semantic functions as long as they belong to a single command. In order to avoid unwanted effects on the environment semantic evaluation starts only when a complete and correct command has been entered by the user, e.g. a nonterminal belonging to part (1) of the AG, the dialogue-grammar is accepted. The attributation is not limited besides in the case of cyclic dependencies. The interpretation starts with functions which have all input parameters instantiated.

\section{References}

[DANG89] Dang, Weidong: Formal Specification of Interactive Languages using Definite Clause Grammar. in: [DERANSART89], pp. 283-291.

[DERANSART89] Deransart, P.; Lorho, B.; Maluszynski, J. (Eds.): PLILP'88, Orleans, France, May 1988, Lecture Notes in Computer Science 348, Berlin(West), 1989.

[LÄMMEL85] Lämmel, U.; Riedewald, G.: RUEGEN-KS - Ein System zur Generierung von Dialogsystemen. Univ. Rostock, Sekt. Informatik, UR-PS-Nr. $9(1985)$.

[OLSEN83] Olsen, D.; Dempsey, E.: Syntax directed Graphical Interaction, ACM SIGPLAN Notices $18(1983) 6$. 